\title{
OPERATIONAL QUADRATURE METHODS FOR WIENER-HOPF INTEGRAL EQUATIONS
}

\author{
P. P. B. EGGERMONT AND CH. LUBICH
}

\begin{abstract}
We study the numerical solution of Wiener-Hopf integral equations by a class of quadrature methods which lead to discrete Wiener-Hopf equations, with quadrature weights constructed from the Fourier transform of the kernel (or rather, from the Laplace transforms of the kernel halves). As the analytical theory of Wiener-Hopf equations is likewise based on the Fourier transform of the kernel, this approach enables us to obtain results on solvability and stability and error estimates for the discretization. The discrete Wiener-Hopf equations are solved by using an approximate Wiener-Hopf factorization obtained with FFT. Numerical experiments with the Milne equation of radiative transfer are included.
\end{abstract}

\section{INTRODUCTION}

The approximate solution of Wiener-Hopf integral equations

$$
x(t)+\int_{0}^{\infty} k(t-\tau) x(\tau) d \tau=f(t), \quad 0<t<\infty,
$$

has received attention from mathematicians of different schools and backgrounds, partly because of direct applications of such equations in mathematical physics, partly because of the insight that the study of these equations gives to the treatment of Fredholm integral equations over 'large' intervals and of dual integral equations, and further because of the rich mathematics that comes along with their theory. Apart from the fundamental article of Krein [18], which gives a complete analytical solution theory of equations (1.1) with absolutely integrable kernel, we mention numerically oriented work of Gohberg and Fel'dman [13], Prössdorf and Silbermann [22, 23], Atkinson [5], Stenger [28], Sloan and Spence [26, 27], Anselone and Baker [1], Chandler and Graham [7], Graham and Mendes [15], Elschner [10], Gähler and Gähler [11], and Anselone and Sloan [3, 4].

In this paper we consider the application of operational quadrature methods to the numerical solution of Wiener-Hopf integral equations, continuing work done in [19] and in our joint paper [9]. These quadrature methods lead to discrete Wiener-Hopf equations

$$
x_{n}+h \sum_{j=0}^{\infty} k(n-j ; h) x_{j}=f_{n}, \quad n=0,1,2, \ldots,
$$

Received by the editor March 29, 1991.

1991 Mathematics Subject Classification. Primary 65R20, 45E10. 
where for a meshwidth $h>0, x_{n}$ is to approximate $x(n h)$, and $k(n ; h)$ are suitably defined quadrature weights. In [19, 111$]$, it was shown for equations (1.1) with real symmetric kernel $k$, that the discretized equation (1.2) arising from an $A$-stable operational quadrature method is uniquely solvable if the continuous equation is so, and for all positive meshwidths the inverses of the discrete Wiener-Hopf operators are bounded in $l^{2}$ by the $L^{2}\left(\mathbb{R}^{+}\right)$operator norm of the continuous inverse.

In the present work the emphasis will be on $l^{\infty}$ error and stability estimates, which become available through results from [9], and we extend our results to nonsymmetric Wiener-Hopf equations. For monotone equations a theory analogous to [19] can be developed. In general, if only the Krein conditions for unique solvability of (1.1) hold, then there are stability restrictions on the meshwidth. Still, for small enough $h$ the discretized Wiener-Hopf operators are shown to have uniformly bounded inverses in all $l^{p}$ spaces $(1 \leq p \leq \infty)$. We also show that exponential decay of both the kernel and the right-hand side in (1.1) yields exponential decay of the discrete solutions, with a mesh-independent rate.

A further topic is the actual solution of the discrete Wiener-Hopf equation (1.2). As a very efficient alternative to the usual procedure of truncating the equation and solving the thus arising Toeplitz system of linear equations, we propose here to use an approximate Wiener-Hopf factorization obtained with FFT. ${ }^{1}$ In the case of exponential decay this allows us to compute the solution of (1.2) to the level of the discretization error with $O\left(h^{-1}|\log h|^{2}\right)$ operations, as $h \rightarrow 0$. Numerical experiments with the Milne equation of radiative transfer illustrate the performance of the methods.

\section{CONDITIONS ON THE TRANSFORMS OF THE KERNEL}

The integral equation $(1.1)$ is considered as an operator equation in $L^{p}\left(\mathbb{R}^{+}\right)$ for $1 \leq p \leq \infty$,

$$
(\mathscr{I}+\mathscr{K}) x=f, \quad f \in L^{p}\left(\mathbb{R}^{+}\right),
$$

where $\mathscr{I}$ is the identity operator, and $\mathscr{K}$ denotes the integral operator in (1.1).

For $k \in L^{1}(\mathbb{R})$, the Krein necessary and sufficient conditions for the invertibility of $\mathscr{I}+\mathscr{K}$ in $L^{p}\left(\mathbb{R}^{+}\right)$are, Krein [18],

$$
\begin{gathered}
1+\hat{k}(\omega) \neq 0 \text { for all } \omega \in \mathbb{R}, \\
\operatorname{index}(1+\hat{k})=\frac{1}{2 \pi} \int_{-\infty}^{\infty} d_{\omega} \arg (1+\hat{k}(\omega))=0,
\end{gathered}
$$

where $\hat{k}(\omega)$ is the Fourier transform of $k$ :

$$
\hat{k}(\omega)=\int_{-\infty}^{\infty} k(t) e^{-i \omega t} d t, \quad \omega \in \mathbb{R} .
$$

Condition (2.2b) says that the closed curve $\{1+\hat{k}(\omega): \omega \in \overline{\mathbb{R}}\}$ does not encircle the origin. Here, $\overline{\mathbb{R}}=\mathbb{R} \cup\{\infty\}$, and $\hat{k}(\infty)=\lim _{\omega \rightarrow \pm \infty} \hat{k}(\omega)=0$ by the Riemann-Lebesgue lemma.

\footnotetext{
${ }^{1}$ The approximate Wiener-Hopf factorization can effectively be used also as a preconditioner in iterative methods for the solution of finite Toeplitz systems of equations. Cf. Chan and Strang [6] who use conjugate gradients with a circulant preconditioner. This application is, however, outside the scope of the present paper.
} 
The Krein conditions are implied by the much stronger monotonicity (or coercivity) condition

$$
\operatorname{Re}(1+\hat{k}(\omega)) \geq \gamma \quad \text { for all } \omega \in \mathbb{R},
$$

where $\gamma$ is some fixed positive number. We note that for real symmetric kernel $k$, conditions (2.2) and (2.4) for some positive $\gamma$ are equivalent, because for such kernels the Fourier transform takes only real values.

Instead of imposing smoothness assumptions on $k$, we will assume that the following sectorial conditions are satisfied: We let $K_{ \pm}(s)$ be the Laplace transforms of the functions $k( \pm t), t>0$, i.e.,

$$
K_{ \pm}(s)=\int_{0}^{\infty} k( \pm t) e^{-s t} d t, \quad \operatorname{Re} s \geq 0
$$

and we assume that there is an angle $\vartheta<\frac{1}{2} \pi$, and positive constants $\alpha$ and $\beta$, such that

$$
\begin{gathered}
K_{+}(s) \text { and } K_{-}(s) \begin{array}{l}
\text { are analytic and bounded } \\
\text { in the sector }|\arg s|<\pi-\vartheta
\end{array} \\
K_{ \pm}(s)-K_{ \pm}(0)=\mathscr{O}\left(s^{\alpha}\right), \quad \text { as } s \rightarrow 0 \text { in the sector } \\
K_{ \pm}(s)=\mathscr{O}\left(s^{-\beta}\right), \text { as } s \rightarrow \infty \text { in the sector. }
\end{gathered}
$$

These conditions can be shown to be equivalent to the following: The kernel halves $k( \pm t)$ are analytic in a sector $|\arg t|<\frac{1}{2} \pi-\vartheta^{\prime}$ with $\vartheta^{\prime}<\frac{1}{2} \pi$, and are bounded there by $|k( \pm t)| \leq$ const $\cdot \min \left\{|t|^{\beta-1},|t|^{-\alpha-1}\right\}$. Hence $k \in L^{1}(\mathbb{R})$, and a weak singularity may occur at 0 . For example, kernels with logarithmic singularity such as the exponential integral $E_{1}$ or the modified Bessel function $K_{0}$, which arise in applications, are included here.

We note for later use that

$$
\hat{k}(\omega)=K_{+}(i \omega)+K_{-}(-i \omega), \quad \omega \in \mathbb{R} .
$$

Remark. Although the above sectorial conditions are certainly much stronger than would be necessary, we have included them here for the following reasons: The approximation theory in [19] is based on sectorial assumptions, and so is a lemma from [9] which is essential for the $l^{\infty}$ and general $l^{p}$ theory (see Lemma 3.1 below). There is no limitation on the obtainable order of approximation of operational quadrature methods under sectorial conditions. In contrast, if the Laplace transforms $K_{ \pm}$are analytic and suitably bounded only in a halfplane, then the operational quadrature methods have to be based on $A$-stable discretizations, and their approximation order cannot exceed 2. See [20] for a nonsectorial approximation theory.

\section{OPERATIONAL QUADRATURE METHODS APPLIED TO WIENER-HOPF EQUATIONS}

As in [19], the quadrature weights $k(n ; h)$ in (1.2) are defined as the coefficients of the generating function, $\mathrm{cf}$. (2.7),

$$
h \sum_{n=-\infty}^{\infty} k(n ; h) \zeta^{n}=K_{+}(\delta(\zeta) / h)+K_{-}\left(\delta\left(\zeta^{-1}\right) / h\right), \quad \zeta \in \mathbb{C},|\zeta|=1
$$


where $\delta(\zeta)$ is given as the quotient of the generating polynomials of a linear multistep method for the solution of differential equations, see e.g., [16],

$$
\sum_{j=0}^{d} \alpha_{j} y_{n+j}=h \sum_{j=0}^{d} \beta_{j} y_{n+j}^{\prime}, \quad n \geq 0,
$$

that is,

$$
\delta(\zeta)=\sum_{j=0}^{d} \alpha_{j} \zeta^{d-j} / \sum_{j=0}^{d} \beta_{j} \zeta^{d-j} .
$$

We assume here that the linear multistep method is consistent of order $M$ and $A(\varphi)$-stable with an angle $\varphi$ greater than $\vartheta$ of (2.6), i.e.,

$$
\begin{aligned}
\delta(\zeta) \text { has neither zeros nor poles on the closed unit disk }|\zeta| & \leq 1 \\
\text { with the exception of a simple zero at } \zeta & =1,
\end{aligned}
$$

The following lemma collects important properties of the quadrature weights. This is a reformulation of Lemma 6.3 in [9] (with $R_{\lambda}$ replaced by $K_{ \pm}$).

Lemma 3.1. Under the conditions (2.6), (3.3) on $k$ and $\delta$ the quadrature weights $k(n ; h)$ satisfy for all $h>0$ and all integer $n$ and $l$

$$
\begin{gathered}
|k(n ; h)| \leq \text { const } \cdot b(|n h|), \\
h \sum_{n=-\infty}^{\infty}|k(n+l ; h)-k(n ; h)| \leq e(|l h|),
\end{gathered}
$$

where $e \in C[0, \infty)$, with $e(0)=0$, is an increasing function, and $b \in L^{1}\left(\mathbb{R}^{+}\right)$ is defined as $b(t)=\min \left\{t^{\beta-1}, t^{-\alpha-1}\right\}$, with $\alpha, \beta$ from (2.6).

We denote the discrete Wiener-Hopf operator in (1.2) by $K_{h}$, so that for $x_{h}=\left\{x_{n}\right\}_{n \geq 0}$ we have

$$
\left[K_{h} x_{h}\right]_{n}=h \sum_{j=0}^{\infty} k(n-j ; h) x_{j}, \quad n \geq 0 .
$$

From Lemma 3.1 we know that $\sup \left\{h \sum_{n=-\infty}^{\infty}|k(n ; h)|: h>0\right\}<\infty$. Consequently, we have for $1 \leq p \leq \infty$

$$
K_{h}: l^{p} \rightarrow l^{p}, \quad \text { with }\left\|K_{h}\right\|_{l^{p}} \leq \text { const },
$$

where the constant is independent of $h>0$.

To get the full order of accuracy $M$, end correction terms have to be added in (1.2). For appropriate correction weights $\kappa(n, j ; h)$ to be determined in $\S 4$ we let the small-rank operator $U_{h}$ be given for a fixed integer $J$ as

$$
\left[U_{h} x_{h}\right]_{n}=h \sum_{j=0}^{J} \kappa(n, j ; h) x_{j}, \quad n \geq 0,
$$


and the Wiener-Hopf integral equation (1.1) is now approximated by the perturbed discrete Wiener-Hopf equation

$$
x_{h}+\left(K_{h}+U_{h}\right) x_{h}=r_{h} f,
$$

where $r_{h}$ denotes the restriction operator

$$
\left[r_{h} f\right]_{n}=f(n h), \quad n \geq 0 .
$$

The end-term correction $U_{h}$ will be constructed in such a way that $\left(K_{h}+U_{h}\right) r_{h} x$ is a "better" approximation to $\mathscr{K} x$.

We are now interested in whether the discrete equation (3.7) has (always) a unique solution $x_{h}$, and in how to estimate the approximation error $x_{h}-r_{h} x$. An elementary error analysis gives

$$
x_{h}-r_{h} x=\left(I+K_{h}+U_{h}\right)^{-1}\left[\left(K_{h}+U_{h}\right) r_{h} x-r_{h} \mathscr{K} x\right] .
$$

So now the question is to show (e.g.) that for appropriate $h_{0}>0$ we have

$$
\begin{gathered}
\sup _{h<h_{0}}\left\|\left(I+K_{h}+U_{h}\right)^{-1}\right\|_{l^{p}}<\infty, \\
h^{1 / p}\left\|\left(K_{h}+U_{h}\right) r_{h} x-r_{h} \mathscr{K} x\right\|_{l^{p}} \leq \mathrm{const} \cdot h^{M},
\end{gathered}
$$

under appropriate smoothness conditions on $x$. In $\S 4$ we discuss $(3.11)$, and (3.10) is considered in $\S \S 5$ and 6 .

\section{QUADRATURE ERROR}

In this section we study the quadrature errors associated with the operational quadrature method. In particular, we are concerned with establishing inequality (3.11) under appropriate conditions on $k, \delta$ and $x$. The estimates follow from Lubich [19] and Eggermont and Lubich [9], cf. also Eggermont [8]. For $M$ an integer we let $W^{\infty}, M\left(\mathbb{R}^{+}\right) \subset L^{\infty}\left(\mathbb{R}^{+}\right)$be the subspace consisting of those functions on $\mathbb{R}^{+}$that together with their derivatives up to order $M$ belong to $L^{\infty}\left(\mathbb{R}^{+}\right)$. The norm on $W^{\infty, M}\left(\mathbb{R}^{+}\right)$is defined as

$$
\|x\|_{W \infty, M}=\sum_{m=0}^{M}\left\|x^{(m)}\right\|_{L^{\infty}} .
$$

Theorem 4.1. Under the assumptions (2.6), (3.3) on the kernel $k$ and the discretization method $\delta$ of consistency order $M$ we have for an appropriate endterm correction $U_{h}$ of the form (3.6) (with $J=M-1$ ) the error bound

$$
\left\|\left(K_{h}+U_{h}\right) r_{h} x-r_{h} \mathscr{K} x\right\|_{l \infty} \leq \mathrm{const} \cdot h^{M} \cdot\|x\|_{W \infty, M}
$$

for all $x \in W^{\infty, M}\left(\mathbb{R}^{+}\right)$. The constant const is independent of the meshwidth $h$ and the function $x$. Moreover,

$$
\left\|U_{h}\right\|_{l \infty} \leq \mathrm{const} \cdot h^{\mu}
$$

where $\mu=\min (1, \beta)$, with $\beta>0$ of $(2.6 \mathrm{c})$.

The end-term correction $U_{h}$ can be chosen as follows [19]: Let $t^{*}>0$ be fixed. The correction weights are then determined such that the quadrature formula is exact for polynomials up to degree $M-1$ over the interval $\left[0, t^{*}\right]$, i.e.,

$$
\left[\left(K_{h}+U_{h}\right) r_{h} p-r_{h} \mathscr{K} p\right]_{n}=0 \text { for } 0 \leq n h \leq t^{*}
$$


for all polynomials $p$ of degree at most $M-1$. This gives a Vandermonde system of linear equations for the computation of the correction weights. For $n h \geq t^{*}$ they are chosen either as $\kappa(n, j ; h)=\left(c_{j}-1\right) k(n-j ; h)$ or as $\left(c_{j}-1\right) k((n-j) h)$, where $c_{j}(j=0,1, \ldots, M-2)$ are the weights of the $M$ th-order Newton-Gregory formula (e.g., $c_{0}=\frac{1}{2}$ for $M=2$, the trapezoidal rule).

Proof of Theorem 4.1. Suppose that the end correction $U_{h}$ has been constructed as described above. We split the quadrature error as

$$
\left[\left(K_{h}+U_{h}\right) r_{h} x-r_{h} \mathscr{K} x\right]_{n}=e_{n}^{+}+e_{-n}^{-},
$$

with

$$
\begin{gathered}
e_{n}^{+}=h \sum_{j=0}^{n} k_{+}(n-j ; h) x(j h)+h \sum_{j=0}^{M-1} \kappa(n, j ; h) x(j h)-\int_{0}^{t} k_{+}(t-\tau) x(\tau) d \tau, \\
e_{n}^{-}=h \sum_{j=-\infty}^{n} k_{-}(n-j ; h) \check{x}(j h)-\int_{-\infty}^{t} k_{-}(t-\tau) \check{x}(\tau) d \tau,
\end{gathered}
$$

where $t=n h, \check{x}(\tau)=x(-\tau), k_{ \pm}(t)=k( \pm t)$, and $k_{ \pm}(n ; h)$ are the coefficients of the generating functions (cf. (3.1))

$$
h \sum_{n=0}^{\infty} k_{ \pm}(n ; h) \zeta^{n}=K_{ \pm}(\delta(\zeta) / h), \quad|\zeta| \leq 1 .
$$

It follows from Lemmas 7.2-7.4 of [9] (which in turn are based on Theorem 3.1 of [19]) that $e_{n}^{+}$is bounded by the right-hand side of (4.1), uniformly in $n$ and $h$. If $x$ (and thus $\check{x}$ ) has compact support, then Lemma 7.4 of [9] shows that

$$
\left|e_{n}^{-}\right| \leq C \cdot h^{M} \cdot\left\|x^{(M)}\right\|_{L^{\infty}} .
$$

For general $x \in W^{\infty, M}\left(\mathbb{R}^{+}\right)$the result then follows by taking smooth truncations of $x$ and using the absolute integrability of the kernel and the uniform absolute summability of the weights.

It remains to verify the bound (4.2). The proof of Corollary 3.2 of [19] shows that $|\kappa(n, j ; h)| \leq C(n h)^{\beta-1}$ for $0 \leq n h \leq t^{*}$, and Lemma 3.1 yields that $|k(n ; h)| \leq C(n h)^{-1-\alpha}$ with $\alpha$ of $(2.6 \mathrm{~b})$ for $n h \geq t^{*}$. In view of the construction of $U_{h}$, these bounds imply (4.2).

We remark that $l^{p}$ estimates (3.11) with $p \neq \infty$ are obtained under the same assumptions on $k$ and $\delta$ and with the same construction of $U_{h}$, if $x \in$ $W^{\infty, M}\left(\mathbb{R}^{+}\right)$with $\left|x^{(M)}(t)\right| \leq$ const $\cdot(1+t)^{-\sigma}$ with $\sigma>\frac{1}{p}$. We omit the details. For $p \neq 1$ one has still

$$
\left\|U_{h}\right\|_{l^{p}} \rightarrow 0 \quad \text { as } h \rightarrow 0 .
$$

We remark further that a similar construction of end-correction weights to restore $M$ th-order convergence can be done for functions $x$ which are not smooth at 0 , but which are known to have an expansion of the form

$$
x(t)=\sum_{i=0}^{I} a_{i} \phi_{i}(t)+\tilde{x}(t)
$$


where $\tilde{x} \in W^{\infty, M}\left(\mathbb{R}^{+}\right)$with $\tilde{x}(0)=\tilde{x}^{\prime}(0)=\cdots=\tilde{x}^{(M-1)}(0)=0$, and the $\phi_{i}$ are given "singular functions", e.g., fractional powers or polynomials multiplied with a logarithm. Details have been worked out by Gienger [12].

\section{SOlVABILITY AND STABILITY IN THE MONOTONE CASE}

In this section we consider the solvability of the discrete equations (1.2) and (3.7) as well as the stability inequality (3.10), when monotonicity is preserved under discretization. For $A$-stable methods, i.e., those with $\operatorname{Re} \delta(\zeta) \geq 0$ for all $|\zeta| \leq 1$, this turns out to be automatically satisfied under the monotonicity condition (2.4). For $A(\varphi)$-stable methods with $\varphi<\frac{1}{2} \pi$ we have to demand that (2.4) hold on a bigger region:

$$
\operatorname{Re}\left(1+K_{+}(s)+K_{-}(\bar{s})\right) \geq \gamma \text { for }|\arg s|=\pi-\varphi,
$$

where $\varphi$ is the same as in (3.3b). Note that for $\varphi=\frac{1}{2} \pi$ this condition is exactly the monotonicity condition (2.4), because of (2.7).

The proof of the following theorem for $p=2$ is in the style of Lubich [19], and from this, the general case follows with results of Eggermont and Lubich [9].

Theorem 5.1. Let $1 \leq p \leq \infty$. Under the conditions (2.6) and (3.3) on the kernel $k$ and the discretization method $\delta$, and assuming the monotonicity condition (5.1), the discrete Wiener-Hopf equation (1.2) has for every $h>0$ a unique solution $x_{h}=\left\{x_{n}\right\}_{n \geq 0} \in l^{p}$ for every $f_{h}=\left\{f_{n}\right\}_{n \geq 0} \in l^{p}$. Moreover, there exists a constant $c$ such that the solution $x_{h}$ satisfies the estimate $\left\|x_{h}\right\|_{l^{p}} \leq c\left\|f_{h}\right\|_{l^{p}}$ uniformly in $h>0$, or equivalently,

$$
\sup _{h>0}\left\|\left(I+K_{h}\right)^{-1}\right\|_{l^{p}} \leq c .
$$

Proof. The first fact to observe is that the Krein conditions for the discrete Wiener-Hopf equation (1.2) are, Krein [18] and (3.1),

$$
\begin{gathered}
a(\zeta) \stackrel{\text { def }}{=} 1+K_{+}(\delta(\zeta) / h)+K_{-}\left(\delta\left(\zeta^{-1}\right) / h\right) \neq 0 \text { for all }|\zeta|=1 \\
\quad \text { index } a \stackrel{\text { def }}{=} \frac{1}{2 \pi} \int_{0}^{2 \pi} d_{\theta} \arg a\left(e^{-i \theta}\right)=0
\end{gathered}
$$

Now consider the function $\Psi(s)=1+K_{+}(s)+K_{-}(\bar{s})$. Since $K_{ \pm}$are analytic in the sector $|\arg s| \leq \pi-\varphi$, it follows that $\operatorname{Re} \Psi$ is harmonic there. Since $\Psi(s) \rightarrow 1$ as $|s| \rightarrow \infty$ in the sector, we may apply the maximum principle, see, e.g., Protter and Weinberger [24, Theorem 2], to $\operatorname{Re} \Psi$ to conclude with the help of (5.1) that for all $s$ in the sector,

$$
\operatorname{Re} \Psi(s) \geq \min \{\operatorname{Re} \Psi(\sigma):|\arg \sigma|=\pi-\varphi\} \geq \gamma .
$$

The $A(\varphi)$-stability condition (3.3b) now shows that $\operatorname{Re} a(\zeta) \geq \gamma$ for all $|\zeta|=1$ and all $h>0$, from which both (5.3a) and (5.3b) follow. We also observe that if $u \in l^{2}$, then by two applications of the Parseval formula, see Stein and Weiss [25], we obtain (with $\hat{u}(\theta)=\sum_{n=0}^{\infty} u_{n} e^{i n \theta}$ )

$$
\begin{aligned}
\operatorname{Re}\left\langle u+K_{h} u, u\right\rangle_{l^{2}} & =\operatorname{Re} \frac{1}{2 \pi} \int_{0}^{2 \pi} a\left(e^{i \theta}\right)|\hat{u}(\theta)|^{2} d \theta \\
& \geq \gamma \frac{1}{2 \pi} \int_{0}^{2 \pi}|\hat{u}(\theta)|^{2} d \theta=\gamma\|u\|_{l^{2}}^{2} .
\end{aligned}
$$


Consequently, for all $u \in l^{2}$ we have $\left\|u+K_{h} u\right\|_{l^{2}} \geq \gamma\|u\|_{l^{2}}$. Since $I+K_{h}$ is invertible by (5.3), this shows that (5.2) holds with $c=1 / \gamma$ for $p=2$. With Lemma 3.1 it then follows from [9, Theorem 4.1] that (5.2) holds for $p=\infty$, and with the discrete analogue of [9, Theorem 8.3] then also for all $p, 1 \leq p \leq \infty$.

Corollary 5.2. Let $1<p \leq \infty$. Under the assumptions (2.6), (3.3), (5.1) on $k$ and $\delta$ there exists an $h_{0}>0$ such that for all $0<h<h_{0}$ the 'corrected' discrete Wiener-Hopf equation (3.7) is uniquely solvable in $l^{p}$ and, moreover,

$$
\sup _{h<h_{0}}\left\|\left(I+K_{h}+U_{h}\right)^{-1}\right\|_{l p}<\infty .
$$

Proof. We write $I+K_{h}+U_{h}=\left(I+K_{h}\right)\left(I+W_{h}\right)$ with $W_{h}=\left(I+K_{h}\right)^{-1} U_{h}$. From Theorem 5.1 and (4.4) it follows that $\left\|W_{h}\right\|_{l p} \rightarrow 0$ as $h \rightarrow 0$, so that there exists an $h_{0}>0$ such that for $0<h<h_{0}$ we have $\left\|W_{h}\right\|_{l^{p}} \leq \frac{1}{2}$. Then $I+W_{h}$ is invertible, and $\left\|\left(I+W_{h}\right)^{-1}\right\|_{l^{p}} \leq 2$, and the corollary follows.

Remark. Note that the choice of $h_{0}$ in Corollary 5.2 is nearly constructive, since it depends on quantities which can be computed. The norm $\left\|U_{h}\right\|$ is available in principle, as is an estimate for $\left\|\left(I+K_{h}\right)^{-1}\right\|$, at least in the 2-norm.

\section{Solvability AND STABILITY UNDER THE 'Plain' KREIN CONDITIONS}

We now consider what happens when $(5.1)$ is replaced by the much weaker Krein conditions $(2.2 \mathrm{a}-\mathrm{b})$. We note here that the combination of an $A(\varphi)$-stable method (with $\varphi<\frac{1}{2} \pi$ ) and the monotonicity condition (2.4) on the half-plane (as opposed to the monotonicity condition (5.1) on the bigger sector) does not appear to give stronger conclusions than the 'plain' Krein conditions do.

Theorem 6.1. Let $1 \leq p \leq \infty$. Under the conditions (2.6) and (3.3) on $k$ and $\delta$, and assuming the Krein conditions (2.2a-b), there exists an $h_{0}>0$ such that for all $0<h<h_{0}$ the discrete Wiener-Hopf equation (1.2) has a unique solution in $l^{p}$ for right-hand sides in $l^{p}$, and

$$
\sup _{h<h_{0}}\left\|\left(I+K_{h}\right)^{-1}\right\|_{l p}<\infty .
$$

Remark 6.2. We note that Corollary 5.2 applies here as well, once Theorem 6.1 has been shown.

Remark 6.3. We note that Theorem 6.1, resp. Corollary 5.2, combined with the estimates of Theorem 4.1 gives us convergence in the $l^{p}$ norm on the whole half-line. For $p<\infty$ this still holds after some form of truncation is considered for the approximate solution of the infinite system (3.7). For $p=\infty$, without any assumptions about a decay of the solution as $t \rightarrow \infty$, we can expect only convergence on compact subsets of $\mathbb{R}^{+}$for the truncated systems, see Anselone and Baker [1], Anselone and Sloan [4], Anselone and Lee [2].

Proof of Theorem 6.1. There are two aspects to Theorem 6.1. One is the invertibility of the operators $I+K_{h}$, for suitably small $h$, the other is the uniform boundedness of the inverses as exemplified by (6.1).

(a) The essential observation in the proof of the invertibility is that for small $h$ the values of $\delta(\zeta) / h$ either lie very close to the imaginary axis, or else are very 
large, so that the estimates $K_{ \pm}(s)=\mathscr{O}\left(s^{-\beta}\right)$ are meaningful. Consequently, we will have that either $a(\zeta)$, see $(5.3 a)$, is very close to $1+\hat{k}(\omega)$ for suitable $\omega$, or $a(\zeta)$ is close to 1 . In either case we have that $a(\zeta) \neq 0$ if $(2.2 \mathrm{a})$ holds. By the same reasoning, under condition $(2.2 \mathrm{~b}), a(\zeta)$ cannot encircle the origin as $\zeta$ runs through the unit circle, so that index $a=0$. Hence, the discrete Krein conditions (5.3) are satisfied for small $h$, and the invertibility in $l^{p}$ follows.

(b) We now consider the uniform boundedness aspect for the case $p=\infty$. Other values of $p$ will be considered later. So we consider equation (1.2) rewritten as

$$
x_{h}+K_{h} x_{h}=f_{h},
$$

where $f_{h} \in l^{\infty}$. By the discussion in part (a), for $h$ small enough this equation has a unique solution $x_{h} \in l^{\infty}$, but we need to bound $\left\|x_{h}\right\|_{l_{\infty}}$ in terms of $\left\|f_{h}\right\|_{l \infty}$. It is helpful to introduce the operator $\pi_{h}: l^{\infty} \rightarrow L^{\infty}$ which carries a sequence $u \in l^{\infty}$ into its piecewise linear interpolant on the mesh $\{n h: n \geq 0\}$ : $\left[\pi_{h} u\right](n h)=u_{n}$ for $n \geq 0$. From (6.2) we have that

$$
\pi_{h} x_{h}+\mathscr{K} \pi_{h} x_{h}-\left(\mathscr{K} \pi_{h}-\pi_{h} K_{h}\right) x_{h}=\pi_{h} f_{h},
$$

and since, by (6.2), $x_{h}=f_{h}-K_{h} x_{h}$, this finally gives that

$$
\pi_{h} x_{h}+\mathscr{K} \pi_{h} x_{h}-\mathscr{E}_{h} x_{h}=g_{h},
$$

where $g_{h}=\pi_{h} f_{h}+\left(\mathscr{K} \pi_{h}-\pi_{h} K_{h}\right) f_{h}$ and

$$
\mathscr{E}_{h}=\left(\pi_{h} K_{h}-\mathscr{K} \pi_{h}\right) K_{h} \text {. }
$$

We note that $\left\|g_{h}\right\|_{l \infty} \leq$ const $\cdot\left\|f_{h}\right\|_{l \infty}$, and we prove below in Lemma 6.4 that $\left\|\mathscr{E}_{h}\right\|_{\left(l^{\infty}, L^{\infty}\right)} \rightarrow 0$ as $h \rightarrow 0$. If we now apply $(I+\mathscr{K})^{-1}$ to both sides of $(6.3)$, and then restrict to the points $n h$, we get that

$$
x_{h}-r_{h}(I+\mathscr{K})^{-1} \mathscr{C}_{h} x_{h}=r_{h}(I+\mathscr{K})^{-1} g_{h},
$$

and since now $\left\|r_{h}(I+\mathscr{K})^{-1} \mathscr{E}_{h}\right\|_{l^{\infty}} \rightarrow 0$, it follows that $\left\|x_{h}\right\|_{l^{\infty}} \leq$ const $\cdot\left\|g_{h}\right\|_{l^{\infty}} \leq$ const $\cdot\left\|f_{h}\right\|_{l_{\infty}}$. This is equivalent to (6.1) for $p=\infty$.

(c) Knowing now that (6.1) holds for $p=\infty$, we claim that it holds for $p=1$ as well. The reason for this is that we may consider the equation adjoint to $(1.1)$, viz., $x+\mathscr{K}^{*} x=f$ :

$$
x(t)+\int_{0}^{\infty} \overline{k(\tau-t)} x(\tau) d \tau=f(t), \quad 0<t<\infty .
$$

The operational quadrature weights associated with this equation $(6.5)$ are given precisely by $h \overline{k(-n ; h)}$, i.e., the operational quadrature approximation to $(6.5)$ is precisely the equation adjoint to (1.2), viz., $x_{h}+K_{h}^{*} x_{h}=f_{h}$ :

$$
x_{n}+h \sum_{j=0}^{\infty} \overline{k(j-n ; h)} x_{j}=f_{n}, \quad n \geq 0 .
$$

The Krein conditions for equations (6.5) and (6.6) are exactly the same as for the original equations (1.1) and (1.2). It follows that if (6.1) holds for $p=\infty$, then it also holds with $K_{h}$ replaced by $K_{h}^{*}$, i.e.,

$$
\sup _{h<h_{0}}\left\|\left(I+K_{h}^{*}\right)^{-1}\right\|_{1 \infty}<\infty,
$$


but this is equivalent to (6.1) for $p=1$. So we now have that (6.1) holds for $p=1$ and $p=\infty$. By the M. Riesz interpolation theorem, Stein and Weiss [25], it follows that (6.1) holds for all $p$ in between as well.

In the proof above we used the following lemma, which is reminiscent of a similar result for compact integral operators.

Lemma 6.4. There holds $\left\|\left(\pi_{h} K_{h}-\mathscr{K} \pi_{h}\right) K_{h}\right\|_{\left(l^{\infty}, L^{\infty}\right)} \rightarrow 0$ as $h \rightarrow 0$.

Proof. Let $u \in l^{\infty}$. Then Lemma 3.1 implies that

$$
\left|\left[K_{h} u\right]_{n}-\left[K_{h} u\right]_{m}\right| \leq d(|n-m| h)\|u\|_{l \infty},
$$

with $d(t)=e(t)+$ const $\cdot t^{\beta}$. Now apply Theorem 4.1 for $M=1$ with $x=$ $\pi_{h} K_{h} u$. Then

$$
\left\|\left(K_{h} r_{h}-r_{h} \mathscr{K}\right) \pi_{h} K_{h} u\right\|_{l^{\infty}} \leq \text { const }\left(h\left\|\pi_{h} K_{h} u\right\|_{W^{\infty, 1}}+h^{\mu}\left\|\pi_{h} K_{h} u\right\|_{L^{\infty}}\right) .
$$

Now

$$
\left\|\left(\pi_{h} K_{h} u\right)^{\prime}\right\|_{L^{\infty}}=\sup _{n} \frac{\left|\left[K_{h} u\right]_{n+1}-\left[K_{h} u\right]_{n}\right|}{h} \leq(d(h) / h)\|u\|_{l^{\infty}},
$$

and $\left\|\pi_{h} K_{h} u\right\|_{L^{\infty}}=\left\|K_{h} u\right\|_{l^{\infty}} \leq$ const $\cdot\|u\|_{l^{\infty}}$, from which it follows that the right-hand side of (6.7) is dominated by $\varepsilon_{h}\|u\|_{l \infty}$ for some $\varepsilon_{h} \rightarrow 0$.

\section{EXPONENTIAL DECAY}

If $e^{\lambda t} k(t) \in L^{1}(\mathbb{R})$ and $e^{\lambda t} f(t) \in L^{\infty}\left(\mathbb{R}^{+}\right)$for some $\lambda>0$, then it is reasonable to expect that $e^{\lambda t} x(t) \in L^{\infty}\left(\mathbb{R}^{+}\right)$as well, with $x(t)$ the solution of (1.1). This is actually true when the following shifted Krein conditions hold, see Krein [18, Theorem 14.1],

$$
\begin{gathered}
1+\hat{k}(\omega+i \lambda) \neq 0 \text { for all } \omega \in \mathbb{R}, \\
\operatorname{index}_{\omega}(1+\hat{k}(\omega+i \lambda))=0,
\end{gathered}
$$

because then (1.1) may be written as

$$
y(t)+\int_{0}^{\infty} e^{\lambda(t-\tau)} k(t-\tau) y(\tau) d \tau=e^{\lambda t} f(t), \quad t \in \mathbb{R}^{+},
$$

and $y(t) \equiv e^{\lambda t} x(t)$. Now (7.1a-b) are the Krein conditions for the WienerHopf equation (7.2), and the conclusion follows. In this section we want to discuss the discrete analogue of this result. It appears that we need to modify the assumption (2.6) on $k$ accordingly, i.e., $e^{\lambda|t|} k(t)$ should satisfy (2.6). This amounts to

$$
\begin{aligned}
& K_{+}(s-\lambda) \text { and } K_{-}(s-\lambda) \text { are analytic and bounded } \\
& \text { in the sector }|\arg s|<\pi-\vartheta \\
& K_{ \pm}(s-\lambda)-K_{ \pm}(-\lambda)=\mathscr{O}\left(s^{\alpha}\right) \text { as } s \rightarrow 0 \text { in the sector } \\
& K_{ \pm}(s-\lambda)=\mathscr{O}\left(s^{-\beta}\right) \text { as } s \rightarrow \infty \text { in the sector. }
\end{aligned}
$$

We then have an exponentially weighted analogue of Lemma 3.1: 
Lemma 7.1. Under the assumptions (7.3) on $k$ and the conditions (3.3) on $\delta$ there exists for every $\mu<\lambda$ an $h_{\mu}>0$ such that the following bounds are valid for $h \leq h_{\mu}$ :

$$
\begin{gathered}
|k(n ; h)| \leq \mathrm{const} \cdot e^{-\mu|n h|} b(|n h|), \quad n=0, \pm 1, \pm 2, \ldots, \\
h \sum_{n=-\infty}^{\infty}\left|e^{\mu|(n+l) h|} k(n+l ; h)-e^{\mu|n h|} k(n ; h)\right| \leq e(|l h|), \quad l=0, \pm 1, \pm 2, \ldots,
\end{gathered}
$$

where $e \in C[0, \infty)$, with $e(0)=0$, is an increasing function, and $b \in L^{1}\left(\mathbb{R}^{+}\right)$ is defined as $b(t)=\min \left\{t^{\beta-1}, t^{-\alpha-1}\right\}$, with $\alpha, \beta$ from (7.3).

The proof of this lemma is analogous to that of Lemma 6.3 in [9], taking now an integration contour shifted to the left by an amount $\lambda$. We omit the details.

We are now ready to quote the theorem on the exponential decay. At this point it is merely a corollary to Theorem 6.1 . It is helpful to introduce the space $l^{p, \mu h}$ of sequences $\left\{x_{n}\right\}_{n \geq 0}$ for which the exponentially weighted sequence $\left\{e^{\mu h n} x_{n}\right\}$ is in $l^{p}$, and whose $l^{p}$ norm defines the $l^{p, \mu h}$ norm of $\left\{x_{n}\right\}$.

Theorem 7.2. Let $1 \leq p \leq \infty$. Under the conditions (7.3) and (3.3) on $k$ and $\delta$, and assuming the shifted Krein conditions (7.1a-b), there exists for every $\mu<\lambda$ an $h_{\mu}>0$ such that for all $0<h<h_{\mu}$ the discrete Wiener-Hopf equation (1.2) has a unique solution in the exponentially weighted space $l^{p, \mu h}$ for right-hand sides in $l^{p, \mu h}$, and

$$
\sup _{h<h_{\mu}}\left\|\left(I+K_{h}\right)^{-1}\right\|_{l p, \mu h}<\infty
$$

\section{APPROXIMATE DISCRETE WIENER-HOPF FACTORIZATION}

In this section we study the approximate solution of the discrete Wiener-Hopf equation

$$
\sum_{j=0}^{\infty} a_{n-j} x_{j}=f_{n}, \quad n \geq 0,
$$

where the sequence $\left\{a_{n}\right\}_{-\infty}^{\infty}$ is absolutely summable, and its symbol

$$
a(\zeta)=\sum_{n=-\infty}^{\infty} a_{n} \zeta^{n}
$$

satisfies the Krein conditions:

$$
\begin{gathered}
a(\zeta) \neq 0 \text { for }|\zeta|=1 \\
\text { index } a(\zeta)=0 .
\end{gathered}
$$

As shown in Krein [18, $\S 13]$, the equation (8.1) then has a unique solution $x=\left\{x_{n}\right\}_{n \geq 0} \in l^{p}$ for every $f=\left\{f_{n}\right\}_{n \geq 0} \in l^{p} \quad(1 \leq p \leq \infty)$, which can be obtained (in theory) from a Wiener-Hopf factorization

$$
a(\zeta)=a_{-}(\zeta) a_{+}(\zeta), \quad|\zeta|=1 .
$$


Here the coefficients $a_{n}^{ \pm}$of $a_{ \pm}(\zeta)$ vanish for negative, resp. nonnegative subscripts $n$. The factors $a_{ \pm}(\zeta)$ are obtained by setting

$$
\log a(\zeta)=: \sum_{n=-\infty}^{\infty} b_{n} \zeta^{n}=\sum_{n=-\infty}^{-1} b_{n} \zeta^{n}+\sum_{n=0}^{\infty} b_{n} \zeta^{n} \equiv b_{-}(\zeta)+b_{+}(\zeta)
$$

and by letting

$$
a_{ \pm}(\zeta):=\exp \left(b_{ \pm}(\zeta)\right)
$$

which clearly yields (8.4). The solution of $(8.1)$ is then given as the coefficient sequence of

$$
\sum_{n=0}^{\infty} x_{n} \zeta^{n} \equiv x(\zeta):=\frac{1}{a_{+}(\zeta)} P_{+}\left(\frac{f(\zeta)}{a_{-}(\zeta)}\right),
$$

where $f(\zeta)=\sum_{n=0}^{\infty} f_{n} \zeta^{n}$, and $P_{+}$denotes the operator that carries an arbitrary Laurent series into its principal part:

$$
P_{+}\left(\sum_{n=-\infty}^{\infty} c_{n} \zeta^{n}\right)=\sum_{n=0}^{\infty} c_{n} \zeta^{n} .
$$

We are now ready to describe the algorithm for the approximate solution of (8.1). This is a finite analogue of the above procedure, which works with sequences of length $2 N$, such as $x=\left\{x_{-N}, \cdots, x_{N-1}\right\}$. We let $F$ denote the discrete Fourier transform of length $2 N$ :

$$
[F x]_{m}=\sum_{n=-N}^{N-1} \omega^{m n} x_{n}, \quad-N \leq m \leq N-1,
$$

where $\omega \equiv e^{\pi i / N}$, with its inverse $F^{-1}$ given by

$$
\left[F^{-1} \hat{x}\right]_{n}=\frac{1}{2 N} \sum_{m=-N}^{N-1} \omega^{-m n} \hat{x}_{m}, \quad-N \leq n \leq N-1 .
$$

Further, we introduce the cutting operator $\Pi_{+}$by setting

$$
\left[\Pi_{+} x\right]_{n}= \begin{cases}x_{n}, & n=0,1, \ldots, N-1 \\ 0, & n=-N, \ldots,-1 .\end{cases}
$$

In the following algorithm all operations on sequences, such as multiplication, division, taking the logarithm, and exponentiation are pointwise.

Algorithm 8.1. Approximate solution of the discrete Wiener-Hopf equation (8.1) Factorization:

$$
\begin{aligned}
& \hat{a}:=\left\{a\left(\omega^{m}\right)\right\}_{m=-N}^{N-1}, \quad \text { where } \omega=e^{\pi i / N}, \\
& \hat{b}:=\log \hat{a} \\
& \hat{b}_{+}:=F \Pi_{+} F^{-1} \hat{b}, \\
& \hat{a}_{+}:=\exp \left(\hat{b}_{+}\right) .
\end{aligned}
$$


Solution procedure:

$$
\begin{aligned}
& \hat{f}:=F f, \quad f=\left\{0,0, \ldots, 0, f_{0}, f_{1}, \ldots, f_{N-1}\right\}, \\
& \hat{u}:=\hat{f} \cdot \hat{a}_{+} / \hat{a} \\
& \hat{u}_{+}:=F \Pi_{+} F^{-1} \hat{u}, \\
& \hat{x}:=\hat{u}_{+} / \hat{a}_{+}, \\
& \left\{x_{n}\right\}_{n=-N}^{N-1} \equiv x:=F^{-1} \hat{x} .
\end{aligned}
$$

Approximate solution: $\left\{x_{0}, x_{1}, \ldots, x_{N-1}, 0,0, \ldots\right\}$.

With the use of FFT, this algorithm requires $\mathscr{O}(N \log N)$ operations. We will give two convergence results for Algorithm 8.1: under minimal assumptions in Theorem 8.2 and for geometrically decreasing sequences in Theorem 8.3. There also the error decreases geometrically with $N$, as will be shown by an analysis based on the aliasing formula for approximate Fourier coefficients and Cauchy's estimates for Laurent series coefficients. Note that after the above 'truncation procedure', we still have uniform convergence on the whole real line, thanks to the exponential decay, cf. Remark 6.3.

To indicate the dependence of the approximate solution on the truncation parameter $N$, we add a superscript $N$ to all sequences appearing in Algorithm 8.1. In particular, the algorithm computes approximate solution values $x_{n}^{N}$ for $0 \leq n \leq N-1$, and $x_{n}^{N}=0$ for $n \geq N$.

Theorem 8.2. Consider the discrete Wiener-Hopf equation (8.1) where $\left\{a_{n}\right\}_{-\infty}^{\infty}$ is absolutely summable and its symbol satisfies the Krein conditions (8.3). For $\left\{f_{n}\right\}_{0}^{\infty} \in l^{1}$, Algorithm 8.1 converges in the $l^{1}$ norm:

$$
\sum_{n=0}^{\infty}\left|x_{n}^{N}-x_{n}\right| \rightarrow 0 \text { as } N \rightarrow \infty .
$$

Proof. We work with the Wiener algebra $\mathscr{W}$ consisting of the functions $c(\zeta)=$ $\sum_{n=-\infty}^{\infty} c_{n} \zeta^{n}$ on the unit circle with absolutely summable Fourier coefficients $\left\{c_{n}\right\}_{-\infty}^{\infty}$, and equipped with the norm $\|c\|_{\mathscr{W}}=\sum_{-\infty}^{\infty}\left|c_{n}\right|$.

For $c(\zeta) \in \mathscr{W}$ we let

$$
\left(I^{N} c\right)(\zeta)=\sum_{n=-N}^{N-1} c_{n}^{N} \zeta^{n}
$$

denote the trigonometric polynomial of order $2 N$ which interpolates $c(\zeta)$ in the points $\omega^{m}=\exp (\pi i m / N),-N \leq m \leq N-1$. Note that the interpolation property is just the relation $\left\{c\left(\omega^{m}\right)\right\}=F\left\{c_{n}^{N}\right\}$. The aliasing formula, see Theorem 13.2a in Henrici [17],

$$
c_{n}^{N}=\sum_{l=-\infty}^{\infty} c_{n+l \cdot 2 N}
$$

shows that $I^{N}: \mathscr{W} \rightarrow \mathscr{W}$ is a bounded linear operator which converges strongly to the identity:

$$
\left\|I^{N} c-c\right\|_{\mathscr{W}} \rightarrow 0 \quad \text { as } N \rightarrow \infty, \text { for all } c \in \mathscr{W}
$$


The same evidently holds for the operator $J^{N}: \mathscr{W} \rightarrow \mathscr{W}$ defined by the partial sum

$$
\left(J^{N} c\right)(\zeta)=\sum_{n=-N}^{N-1} c_{n} \zeta^{n} .
$$

With these preparations, we can reformulate Algorithm 8.1 in the following way:

Factorization :

$$
a_{+}^{N}(\zeta)=\exp \left(P_{+} I^{N} \log a(\zeta)\right)
$$

Solution procedure :

$$
x^{N}(\zeta)=I^{N}\left(\frac{1}{a_{+}^{N}(\zeta)} P_{+} I^{N}\left(\frac{\left(J^{N} f\right)(\zeta) a_{+}^{N}(\zeta)}{a(\zeta)}\right)\right) .
$$

This gives $x^{N}(\zeta)=\sum_{n=-N}^{N-1} x_{n}^{N} \zeta^{n}$ of Algorithm 8.1.

By the Wiener-Levy theorem, the Krein conditions (8.3) imply that $\log a(\zeta)$ and $1 / a(\zeta)$ are again in $\mathscr{W}$. Moreover, $P_{+}$and exp: $\mathscr{W} \rightarrow \mathscr{W}$ are continuous operators. Together with the strong convergence of $I^{N}$ and $J^{N}$ to the identity, this shows that $x^{N}$ converges in $\mathscr{W}$ to $x$ of (8.7). This is the desired result.

We now turn to geometrically decreasing sequences.

Theorem 8.3. Consider the discrete Wiener-Hopf equation (8.1) whose symbol $a(\zeta)$ is assumed analytic and without zeros in the annulus $\rho \leq|\zeta| \leq 1 / \rho(0<$ $\rho<1)$, and of index 0 . The right-hand side is assumed to satisfy $\left|f_{n}\right| \leq c \rho^{n}$ for $n \geq 0$. Then there are constants $\frac{1}{4} \leq \alpha<1$ and $C<\infty$ such that the error of Algorithm 8.1 is bounded by

$$
\left|x_{n}^{N}-x_{n}\right| \leq \frac{C}{\left(1-\rho^{\alpha}\right)^{2}} \rho^{\alpha(N+n)}, \quad n \geq 0,
$$

provided that $N$ is so large that $\rho^{\alpha N} \leq c^{\prime}\left(1-\rho^{\alpha}\right)^{2}$. The constant $C$ can be chosen to depend only on $c, c^{\prime}$, and on a bound of $|\log a(\zeta)|$ in the annulus.

Proof. We consider Algorithm 8.1 again in the reformulation (8.16). We thus have to study approximation properties of $I^{N}$ and $J^{N}$, and boundedness of the operator $P_{+}$, when they are applied to functions analytic in an annulus. Suppose for this that the Laurent series $c(\zeta)=\sum_{n=-\infty}^{\infty} c_{n} \zeta^{n}$ satisfies

$$
|c(\zeta)| \leq M, \quad \rho \leq|\zeta| \leq 1 / \rho .
$$

The aliasing formula (8.13), combined with the Cauchy estimates

$$
\left|c_{n}\right| \leq M \rho^{|n|} \quad \text { for all } n
$$

gives

$$
\left|c_{n}^{N}-c_{n}\right| \leq M \cdot\left(\rho^{n}+\rho^{-n}\right) \frac{\rho^{2 N}}{1-\rho^{2 N}}, \quad-N \leq n \leq N-1,
$$

and consequently for $0<\alpha<1$ and all $\zeta$ with $\rho^{1-\alpha} \leq|\zeta| \leq 1 / \rho^{1-\alpha}$,

$$
\begin{gathered}
\left|I^{N} c(\zeta)-c(\zeta)\right| \leq 2 M \cdot \frac{\rho^{\alpha N}}{1-\rho^{\alpha}}, \\
\left|P_{+} I^{N} c(\zeta)-P_{+} c(\zeta)\right| \leq M \cdot \frac{\rho^{\alpha N}}{1-\rho^{\alpha}} .
\end{gathered}
$$


Similarly, we have in this annulus also

$$
\left|J^{N} c(\zeta)-c(\zeta)\right| \leq M \cdot \frac{\rho^{\alpha N}}{1-\rho^{\alpha}} .
$$

Repeated use of these estimates in (8.16) leads to the stated result for $n \leq N-1$. The result for $n \geq N$ follows by applying the Cauchy estimates to $x(\zeta)$ of (8.7).

Remark. Theorems 8.2 and 8.3 remain valid for the following two modifications of Algorithm 8.1:

- Instead of taking $b_{+}:=\Pi_{+} b$ in the third step of Algorithm 8.1, one might take a symmetric splitting $b_{+}=\left\{b_{n}^{+}\right\}_{n=-N}^{N-1}$ with

$$
b_{n}^{+}:= \begin{cases}b_{n}, & n=1, \ldots, N-1, \\ \frac{1}{2} b_{n}, & n=0,-N, \\ 0, & n=-(N-1), \ldots,-1 .\end{cases}
$$

For a real symmetric sequence $\left\{a_{n}\right\}$, this has the advantage that then $\hat{a}=\left|\hat{a}_{+}\right|^{2}$ in the modified Algorithm 8.1.

- If the sequence $\left\{a_{n}\right\}$ is known rather than its symbol $a(\zeta)$, then one can modify the beginning of Algorithm 8.1:

$$
\begin{aligned}
& a:=\left\{a_{n}\right\}_{n=-N}^{N-1}, \\
& \hat{a}:=F a .
\end{aligned}
$$

\section{AsYMPTOTIC WORK ESTIMATE}

We now come to the application of Algorithm 8.1 to the discretized WienerHopf equation (1.2), which by (3.1) has the symbol

$$
a(\zeta)=1+K_{+}(\delta(\zeta) / h)+K_{-}\left(\delta\left(\zeta^{-1}\right) / h\right) .
$$

When one applies Algorithm 8.1 to equation (1.2), there is a surprising feature: The quadrature weights are never computed. In the case of exponential decay as studied in $\S 7$, we can use Theorem 8.2 to obtain an asymptotic bound on the computational work needed to compute the solution of (1.2) to the level of the discretization error.

Theorem 9.1. Under the assumptions of Theorem 7.2, the computation of the solution of the discretized Wiener-Hopf equation (1.2) (or of the 'corrected' equation (3.7)) with an error bounded in $l^{\infty}$ by const $\cdot h^{M}$, requires a number of operations proportional to

$$
h^{-1}|\log h|^{2} \quad \text { as } h \rightarrow 0 .
$$

Proof. With $a(\zeta)$ of $(9.1)$, we may choose $\rho=e^{-\lambda h / 2}$ in Theorem 8.3 , to get

$$
\left|x_{n}^{N}-x_{n}\right| \leq \frac{C}{(\lambda h)^{2}} e^{-\alpha \lambda h(N+n)}, \quad n \geq 0,
$$


for the error introduced by Algorithm 8.1, with a constant $C$ which is independent of the meshwidth $h$. The right-hand side in (9.2) is bounded by const $\cdot h^{M}$ if $N$ is chosen to satisfy

$$
N \geq \frac{c M}{\lambda h}\left|\log \lambda^{2} h^{M+2}\right|
$$

for some sufficiently large constant $c$. Since Algorithm 8.1 requires $O(N \log N)$ operations, this gives the desired work estimate for equation (1.2).

For the small-rank perturbation (3.7) one can use the Sherman-MorrisonWoodbury formula, Golub and Van Loan [14, p. 3], which requires the solution of discrete Wiener-Hopf equations (1.2) for $J+1$ (with $J$ of (3.6)) different right-hand sides which are again exponentially decaying if the correction weights are chosen as in $\S 4$. The result follows as before.

\section{NUMERICAL EXPERIMENTS}

In this section we report on numerical results for the Milne equation of the theory of radiation, whose homogeneous version was already studied by Wiener and Hopf, see also Krein [18, §15]:

$$
x(t)-\frac{\alpha}{2} \int_{0}^{\infty} E_{1}(|t-\tau|) x(\tau) d \tau=f(t), \quad t \geq 0
$$

where $\alpha<1$ is a parameter, and $E_{1}(t)=\int_{t}^{\infty} e^{-\tau} / \tau d \tau$ is the exponential integral, whose Laplace transform is $s^{-1} \log (1+s)$. (The kernel has a logarithmic singularity at 0 , and decays exponentially.)

We give numerical results for $\alpha=0.9$, for two choices of $f$. In our first example we take $f(t) \equiv 1$. For the numerical treatment this presents the minor inconvenience that $f$ does not tend to 0 as $t$ approaches infinity. However, we can modify our equations similarly to the modified finite section approach of Sloan and Spence [27]. Taking the limit $t \rightarrow \infty$ in equation (10.1) (which will be justified in a minute), we have

$$
x(\infty)-\frac{\alpha}{2} \int_{-\infty}^{\infty} E_{1}(|\tau|) x(\infty) d \tau=f(\infty)
$$

i.e.,

$$
x(\infty)=\frac{f(\infty)}{1-\alpha} .
$$

Hence, $x^{0}(t)=x(t)-x(\infty)$ satisfies equation (10.1) with the right-hand side

$$
f^{0}(t)=f(t)-x(\infty)+\frac{\alpha}{2} \int_{-\infty}^{t} E_{1}(|\tau|) d \tau \cdot x(\infty)
$$

which in our case is the exponentially decaying function

$$
f^{0}(t)=\frac{\alpha}{2(1-\alpha)}\left(\int_{0}^{t} E_{1}(\tau) d \tau-1\right) .
$$

Conversely, since the solution $x^{0}(t)$ of equation (10.1) with right-hand side (10.3) is known to decay exponentially (cf. $\S 7$ ), taking the limit in (10.1) is 
justified a posteriori, and $x(t)=x^{0}(t)+x(\infty)$ is the unique bounded solution of $(10.1)$ with $f(t) \equiv 1$.

We have applied the operational quadrature method based on the secondorder backward differentiation formula $\delta(\zeta)=\frac{3}{2}-2 \zeta+\frac{1}{2} \zeta^{2}$. The correction weights (3.6) have been chosen so that constants are integrated exactly on an interval near 0 , i.e.,

$$
h \kappa(n, 0 ; h)=\int_{0}^{n h} E_{1}(\tau) d \tau-h \sum_{j=0}^{n} k(n-j ; h) \quad \text { for } 0 \leq n h \leq 2,
$$

and

$$
\kappa(n, 0 ; h)=-\frac{1}{2} E_{1}(n h) \text { for } n h>2 .
$$

It is only in $(10.4 \mathrm{a})$ that the quadrature weights $k(n ; h)$ as defined by $(3.1)$ are needed. These were computed as described in $[19, \S 7]$.

We have used Algorithm 8.1 with

$$
a(\zeta)=1-\alpha \operatorname{Re} \frac{h}{\delta(\zeta)} \log \left(1+\frac{\delta(\zeta)}{h}\right)
$$

for the solution of the discrete Wiener-Hopf equations

$$
\begin{aligned}
& u_{n}-\frac{\alpha}{2} h \sum_{j=0}^{\infty} k(n-j ; h) u_{j}=f^{0}(n h), \quad n \geq 0, \\
& v_{n}-\frac{\alpha}{2} h \sum_{j=0}^{\infty} k(n-j ; h) v_{j}=-\frac{\alpha}{2} h \kappa(n, 0 ; h), \quad n \geq 0 .
\end{aligned}
$$

The solution of the rank-1 perturbed Wiener-Hopf equation

$$
x_{n}^{0}-\frac{\alpha}{2} h \sum_{j=0}^{\infty} k(n-j ; h) x_{j}^{0}-\frac{\alpha}{2} h \kappa(n, 0 ; h) x_{0}^{0}=f^{0}(n h), \quad n \geq 0,
$$

is then given by the Sherman-Morrison formula, see [14] :

$$
x_{n}^{0}=u_{n}-\frac{u_{0} v_{n}}{1+v_{0}} \text {. }
$$

Finally, we have set

$$
x_{n}=x_{n}^{0}+x(\infty)
$$

as an approximation of $x(n h)$.

In Figure 1 (next page) we give the numerical solutions obtained for $h=1 / 32$ and $N h=1,2,4,8,16$, where $N$ is the truncation parameter in Algorithm 8.1 .

In Figures 2 and 3 (next page) we show the relative errors of the method in dependence of the meshwidth $h$ at $t=0$ (where the maximum error occurs) and at $t=4$. Here we have always used $N h=32$ in Algorithm 8.1. The solid lines indicate the errors of $x_{n}$ as constructed above, the dashed lines give the errors of the method without end-term correction, i.e., of $u_{n}+x(\infty)$, with $u_{n}$ given as the solution of $(10.6)$. We note that for the present problem with 


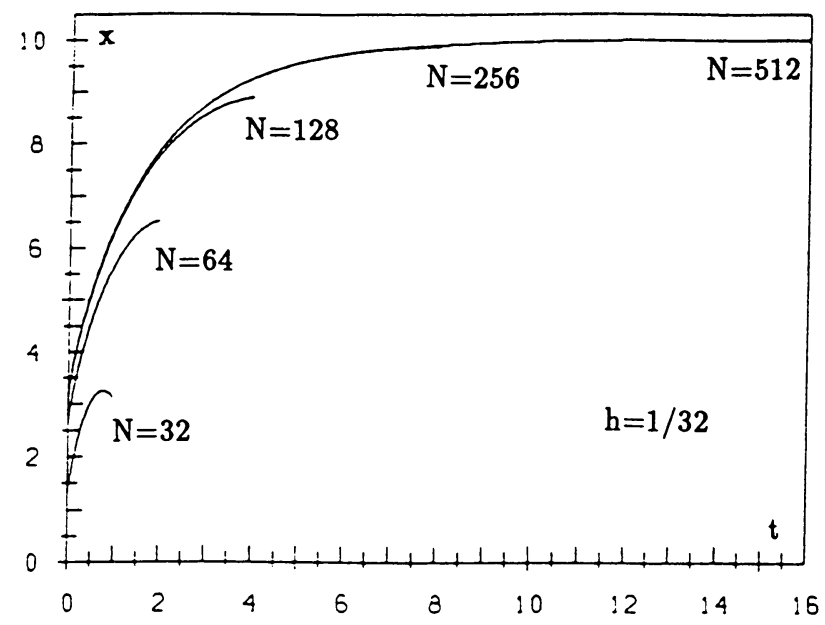

Figure 1. Solution curves
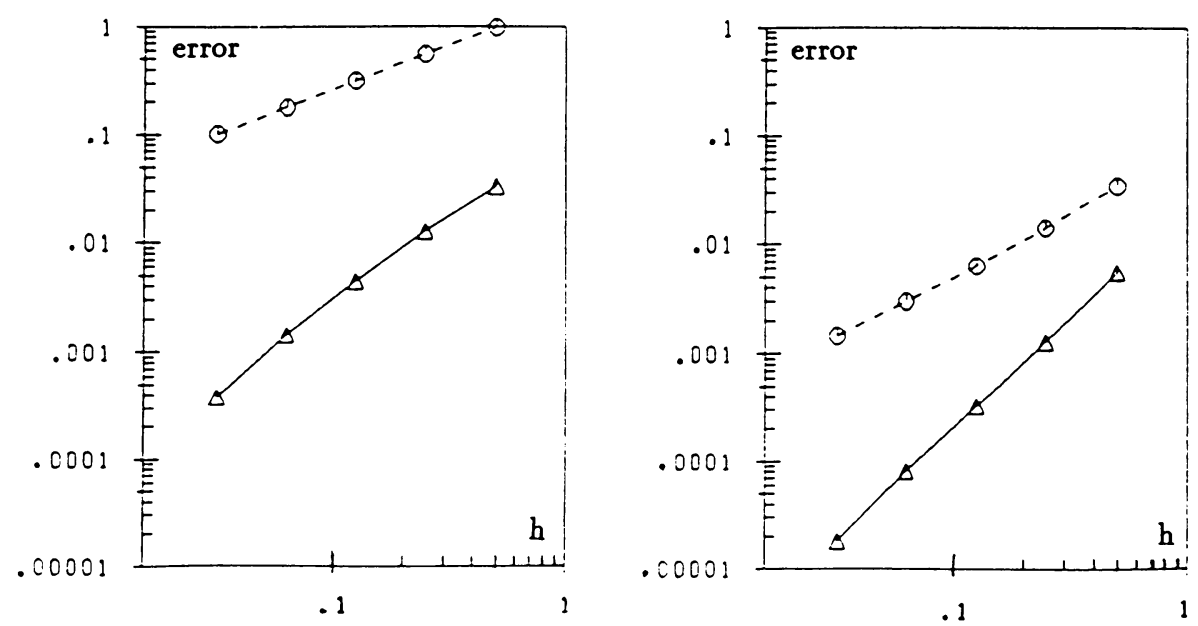

FIGUREs 2 and 3. Error vs. meshwidth at $t=0$ and $t=4$

$f(t) \equiv 1$ the value $x(0)$ is known analytically, see Krein [18, formula (8.9)]: $x(0)=(1-\alpha)^{-1 / 2}$. The reference value at $t=4$ has been obtained numerically, using various discretization methods with small meshwidths.

As a second example, which shows the behavior of the method for nonsmooth data, we have chosen

$$
f(t)= \begin{cases}1, & 0 \leq t \leq 8 \\ 0, & t>8\end{cases}
$$

Figure 4 shows the numerical results obtained with $h=\frac{1}{8}, \frac{1}{16}, \frac{1}{32}$, and $N h=32$. 


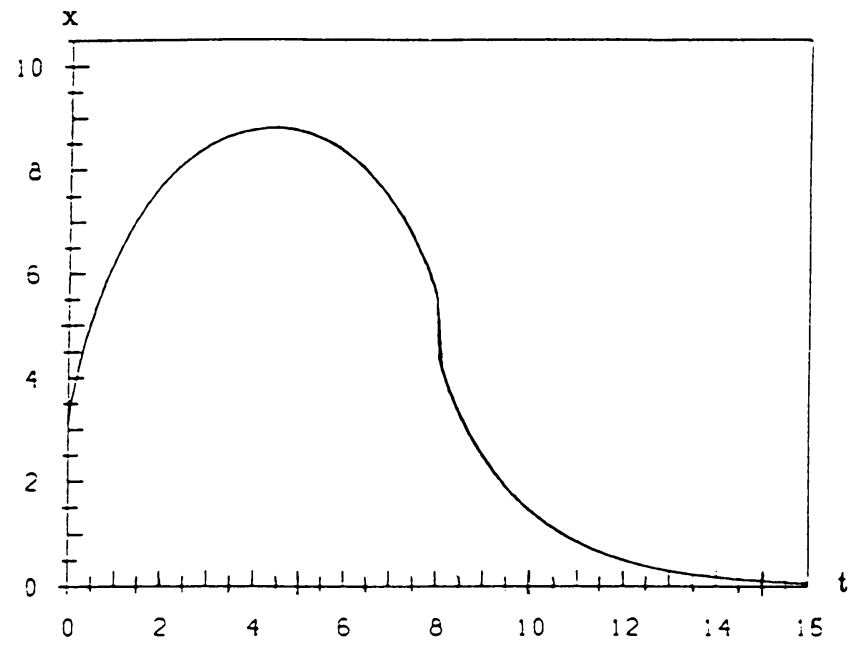

FiguRE 4. Solution curves of second example

\section{BIBLIOGRAPHY}

1. P. M. Anselone and C. T. H. Baker, Error bounds for integral equations on the half line, J. Integral Equations Appl. 1 (1988), 321-341.

2. P. M. Anselone and J. W. Lee, On nonlinear convolution equations on the half line, J. Integral Equations Appl. 4 (1992), 1-14.

3. P. M. Anselone and I. H. Sloan, Integral equations on the half line II: the Wiener-Hopf case, J. Integral Equations Appl. 1 (1988), 203-225 .

4. __ Spectral approximations for Wiener-Hopf operators, J. Integral Equations Appl. (to appear)

5. K. E. Atkinson, The numerical solution of integral equations on the half line, SIAM J. Numer. Anal. 6 (1969), 375-397.

6. R. H. Chan and G. Strang, Toeplitz equations by conjugate gradients with circulant preconditioner, SIAM J. Sci. Statist. Comput. 10 (1989), 104-119.

7. G. A. Chandler and I. G. Graham, The convergence of Nyström methods for Wiener-Hopf equations, Numer. Math. 52 (1988), 345-364.

8. P. P. B. Eggermont, On the quadrature error in operational quadrature methods for convolutions, Numer. Math. 62 (1992), 35-48.

9. P. P. B. Eggermont and Ch. Lubich, Uniform error estimates of operational quadrature methods for nonlinear convolution equations on the half-line, Math. Comp. 56 (1991), 149176.

10. J. Elschner, On spline collocation for convolution equations, Integral Equations Operator Theory 12 (1989), 486-510.

11. S. Gähler and W. Gähler, Quadrature methods for the solution of integral equations of the second kind on the half line, Math. Nachr. 140 (1989), 321-346.

12. G. Gienger, On convolution quadratures and their application to Fredholm integral equations, Mitt. Math. Sem. Giessen 186 (1988), 1-120.

13. I. C. Gohberg and I. A. Fel'dman, Convolution equations and projection methods for their solution, Transl. Math. Monos., vol. 41, Amer. Math. Soc., Providence, RI, 1974.

14. G. H. Golub and C. F. Van Loan, Matrix computations, 2nd ed., The Johns Hopkins University Press, Baltimore, 1989.

15. I. G. Graham and W. R. Mendes, Nyström-product integration for Wiener-Hopf equations with applications to radiative transfer, IMA J. Numer. Anal. 9 (1989), 261-284. 
16. E. Hairer, S. P. Nørsett and G. Wanner, Solving ordinary differential equations I: Nonstiff problems, Springer-Verlag, Berlin, 1987.

17. P. Henrici, Applied and computational complex analysis, vol. 3, Wiley, New York, 1986.

18. M. G. Krein, Integral equations on a half-line with kernel depending upon the difference of the arguments, Amer. Math. Soc. Transl. (2) 22 (1962), 163-286.

19. Ch. Lubich, Convolution quadrature and discretized operational calculus. I and II, Numer. Math. 52 (1988), 129-145 and 413-425.

20. Ch. Lubich, Convolution quadrature and Hille-Phillips operational calculus, Appl. Numer. Math. 9 (1992), 187-199.

21. B. Noble, The numerical solution of nonlinear integral equations and related topics, Nonlinear Integral Equations (P. M. Anselone, ed.), Univ. of Wisconsin Press, Madison, WI, 1964.

22. S. Prössdorf and B. Silbermann, Projektionsverfahren und die näherungsweise Lösung singulärer Gleichungen, Teubner, Leipzig, 1977.

23. $\underset{1}{1}$, Numerical analysis for integral and related operator equations, Birkhäuser, Basel, 1991.

24. M. H. Protter and H. F. Weinberger, Maximum principles in differential equations, PrenticeHall, Englewood Cliffs, NJ, 1967.

25. E. Stein and G. Weiss, Introduction to Fourier analysis on Euclidean spaces, Princeton Univ. Press, Princeton, NJ, 1971.

26. I. H. Sloan and A. Spence, Projection methods for integral equations on the half line, IMA J. Numer. Anal. 6 (1986), 153-172.

27. __ Integral equations on the half-line: a modified finite section approximation. Math. Comp. 47 (1986), 589-595.

28. F. Stenger, The approximate solution of Wiener-Hopf integral equations, J. Math. Anal. Appl. 37 (1972), 687-724.

19716

Department of Mathematical Sciences, University of Delaware, Newark, Delaware

E-mail address: eggermon@brahms.udel.edu

INSTitut Für ANGewandte Mathematik und Statistik, Universität WÜRzburg, Am HUbLAND, D-W-8700 WÜRZBURG, GERMANY

E-mail address: lubich@mathematik.uni-wuerzburg.de 